\title{
Completely semi-stable trees
}

\author{
D.A. Holton
}

Completely semi-stable trees are characterised by the complete absence of three types of subtrees.

\section{Introduction}

All graphs $G$ are on a finite set of vertices $V$, and have no loops, multiple edges or directed edges. For graph concepts used here see [1], and for extra permutation group concepts see [7].

A graph $G$ is semi-stable, [3], if there exists a vertex $v \in V$ such that $\Gamma(G)_{v}=\Gamma\left(G_{v}\right)$, where $\Gamma(G)$ is the automorphism group of $G$. In such a case $G$ is said to be semi-stable at $v$. From [3] we see that all regular graphs are semi-stable, and clearly they must be semi-stable at every vertex. Similarly in [4] it is shown that $P_{m} \times P_{n}$ is semi-stable at every vertex provided

(i) neither $m$ nor $n$ is 1 ,

(ii) if $m=2$ then so is $n$, and vice-versa, and

(iii) $m$ and $n$ are not both equal to 3 ,

where $P_{r}$ is the path on $r$ vertices.

Graphs which are semi-stable at every viertex we call completely semistable. We now consider which trees are completely semi-stable.

\section{Completely semi-stable trees}

In the remainder of this paper $T$ is a tree on a vertex set $V$. 
The following characterisation of semi-stability was proved in [6].

THEOREM 1. G is semi-stable at $v$ if and only if $v$ is adjacent to alz vertices in a fixed block of $\Gamma\left(G_{v}\right)$.

Now it is clear that if we wish to build up a semi-stable tree $T$ from a subforest $T_{v}$, then the new vertex $v$ can be adjacent to exactly one vertex from each of the trees of the subforest. (Obviously if we join $v$ to more than one vertex of a given tree we produce a cycle.) So the fixed block of $\Gamma\left(T_{v}\right)$ to which $v$ is joined must consist of a union of single vertices from each tree of the subforest, and these vertices then must be fixed vertices in the automorphism group of the tree to which they belong. Hence if one of the trees of the subforest $T_{v}$ contains no fixed vertices, then $T$ is not semi-stable at $v$.

LEMMA 1. If $T_{v}$ contains a component with no fixed vertices, then this component is a bicentral tree of the type shown in Figure 1 , and $T$ is not semi-staile at $v$.

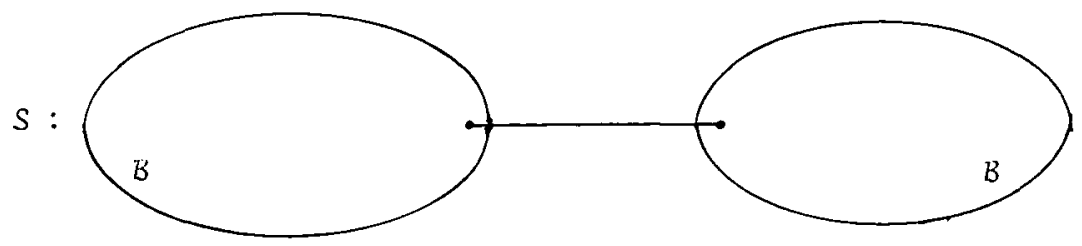

Figure 1

Proof. If a tree $S$ has no fixed vertices then it must be bicentral, and the branches attached to each centre vertex must be the same. The argument used prior to the statement of the Lemma then gives the required result. And in a similar way

LEMMA 2. If $T$ is of the form shown in Figure 2, then $T$ is not semi-stable at $v$.

Proof. In Figure 2, $A, B$, and $C$, are all subtrees of $T$. The vertices $w_{1}$ and $x_{1}, w_{2}$ and $x_{2}$ correspond in the two copies of $B$. clearly $w_{1}$ and $x_{1}$ are in the same fixed block of $\Gamma\left(T_{v}\right)$. 


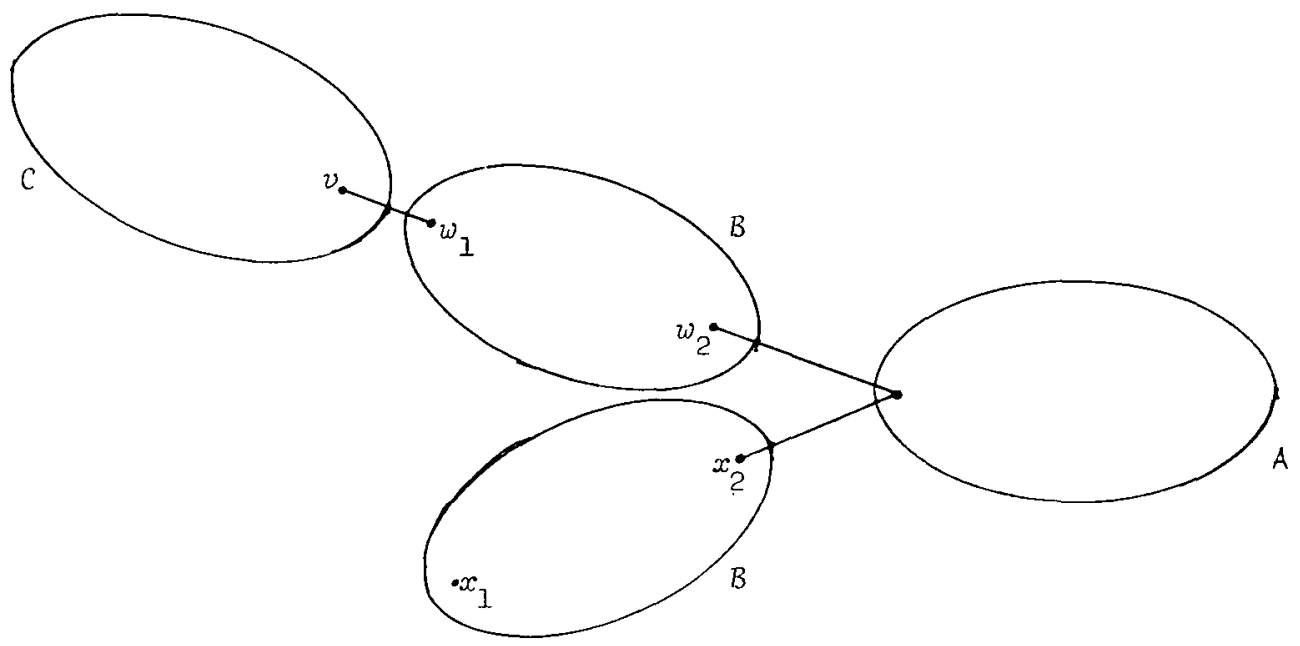

Figure 2

LEMMA 3. If $T$ is as in Figure 3, where $v$ is not necessarily fixed in $\Gamma(T)$, but $B_{1}$ together with $v$ and rooted at $v$ is not isomorphic to $B_{2}$ together with $v$ and rooted at $v$, whereas $\left(B_{1}\right)_{v}=\left(B_{2}\right)_{v}$, then $T$ is not semi-stable at $v$.

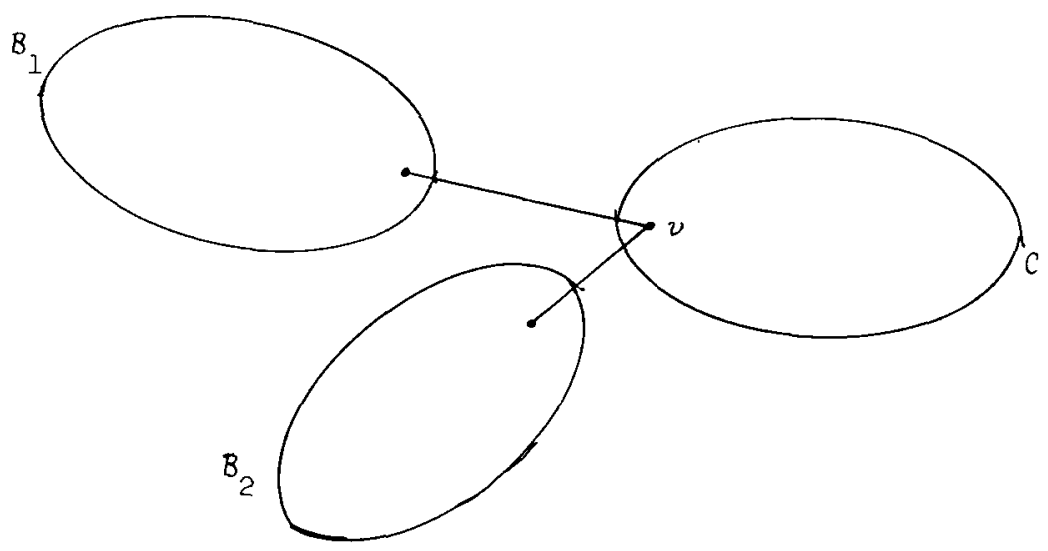

Figure 3

Proof. Clearly removing $v$ introduces extra automorphisms.

Specific examples of trees with each type of inadmissable subtree are 
giver, in order, in Figure 4.

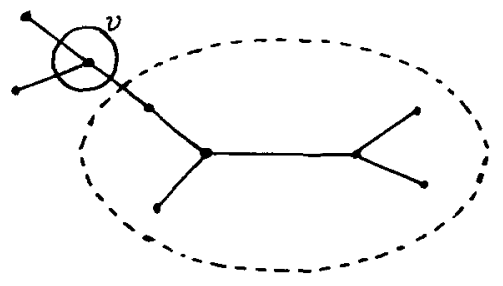

(a) - Lemma 1

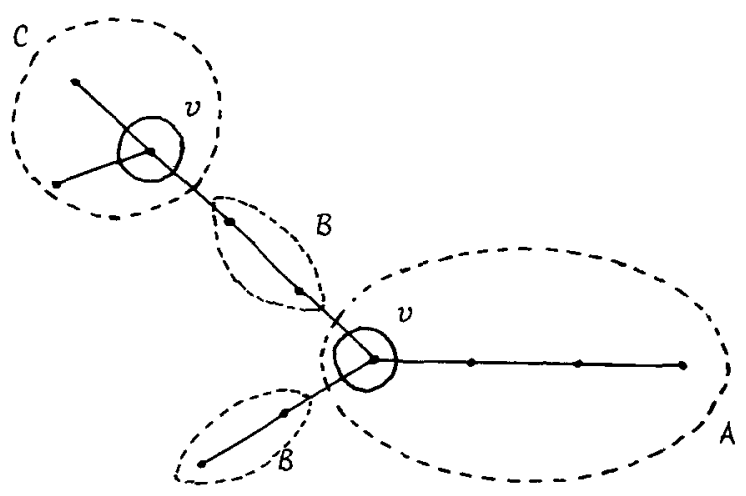

(b) - Lemma 2

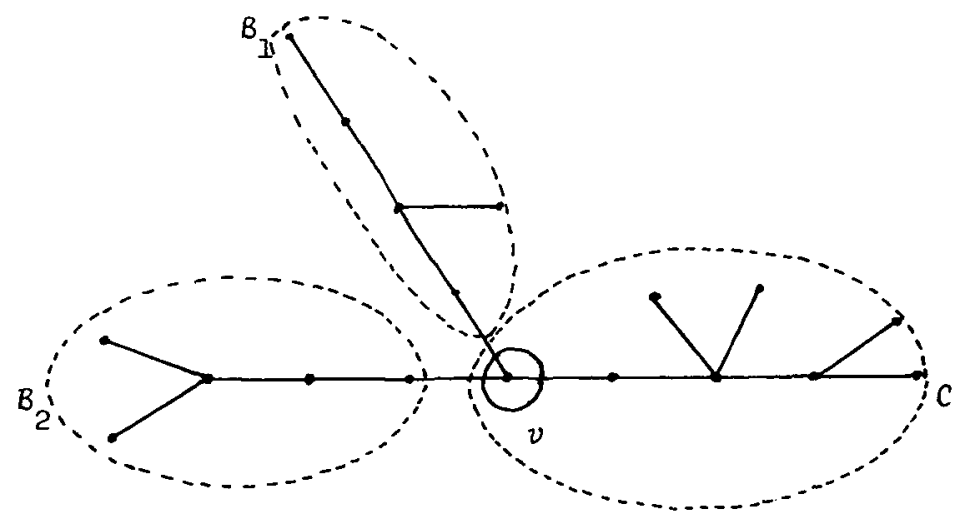

(c) - Lemma 3

Figure 4

The main theorem can now be stated. 
THEOREM 2. $T$ is completely semi-stable if and only if

(i) there is no vertex $v$ in $V$ such that $T_{v}$ contains a component of the type shown in Figure 1 ,

(ii) $T$ is not of the form of Lemma 2, and

(iii) $T$ is not of the form of Lemma 3 .

Proof. $\Leftrightarrow$ follows from Lemmas 1,2 and 3.

$\Leftrightarrow$. Assume there exists a vertex $v$ at which $T$ is not semistable. Then there exists an automorphism $g$ in $\Gamma\left(T_{v}\right)$, which does not occur in $\Gamma(T)_{v}$. (Since in general $\Gamma\left(T_{v}\right) \leq \Gamma\left(T_{v}\right)$ - see [3].) Let $w$ be adjacent to $v$ in $T$ and assume $w^{g} \neq w$. Such a vertex must exist by Theorem 1 .

(1) If $w$ and $w^{g}$ are in the same component of $T_{v}$ and this component, which is a tree, has no fixed vertices under the action of $g$, then the subtree contradicts $(i)$. So assume that $x$ is the nearest fixed vertex to $w$ in the same component of $T_{v}$ as $w$ and $w^{g}$. From elementary properties of automorphisms, the branch of this component which contains $w$, when rooted at $x$, is isomorphic to the branch of the component which contains $w^{g}$, when rooted at $x$. Hence (ii) is contradicted.

(2) Suppose then $w$ and $w^{g}$ are in different components $B_{1}, B_{2}$ of $T_{v}$. Since $g \in \Gamma\left(T_{v}\right)$ then $B_{1}=B_{2}$.

(a) If $w$ is not fixed in $\Gamma\left(B_{1}\right)$ then there exists $h \in \Gamma\left(B_{1}\right) \leq \Gamma\left(T_{v}\right)$ such that $w^{h} \neq w$, and $w^{h} \in B_{1}$. Applying the argument of (1) we obtain a contradiction. (h $\leqslant \Gamma(T)$ since otherwise $w, v$ and $w^{h}$ form a cycle in $T$.)

(b) If $w$ is fixed in $\Gamma\left(B_{1}\right)$ then $w^{9}$ is fixed in $\Gamma\left(B_{2}\right)$. Now either $\underline{b}_{1},\left(B_{1}\right.$ together with $v$ and rooted at $\left.v\right)$ and $\underline{B}_{2},\left(B_{2}\right.$ 
together with $v$ and rooted at $v$ ), are isomorphic or they are not. If they are not then ( $i$ ii) is contradicted. If they are then the non semistability at $v$ is caused by some vertex adjacent to $v$, other than $w$.

Considering all vertices adjacent to $v$ in $T$ in order via ( 1 ) and (2), we either produce a contradiction of (i), (ii) or (iii), or we find that $T$ is semi-stable at $v$. But this is a contradiction. Hence the theorem holds.

\section{Stable graphs}

In [5] the concept of stable graphs was introduced. $G$ is stable if there exists a sequence of all the vertices of $V,\left\{v_{1}, v_{2}, \ldots, v_{n}\right\}$, such that $\Gamma\left(G v_{1} v_{2} \ldots v_{k}\right)=\Gamma(G) v_{1} v_{2} \ldots v_{k}$ for $k=1,2,3, \ldots, n$. It was shown in [2], that a tree $T$ is stable if and only if $\Gamma(T)$ contains a transpositipn.

THEOREM 3. If $T$ is completely semi-stable then $T$ is stable.

Proof. $T$ is stable if and only if it contains a bunch ([2]). A bunch is a set of $r$ (>I) vertices of degree one adjacent to a common vertex.

Suppose $T$ has no bunch. Then it must have a vertex $x$ of degree 2 adjacent to an end vertex $y$ (Figure 5). (This arises since $|V|$ is finite. If the degree of $x$ is more than 2 , move along the tree away from $w$ to the next penultimate vertex. If this has degree greater than 2 , continue away from this vertex over edges not so far traversed. Eventually we must reach a penultimate vertex of degree 2 .)

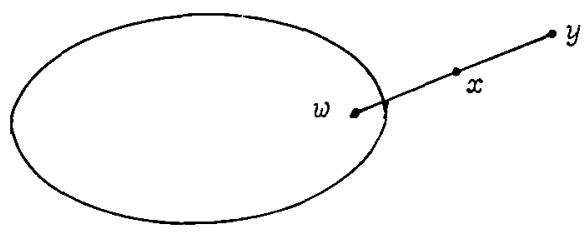

Figure 5

Since $x$ exists as in Figure 5 consider $T_{w}$. This contains an inadmissable bicentral subtree on the vertex set $\{x, y\}$, and so $T$ is 
not semi-stable at $w$ - a contradiction.

However $T$ stable does not imply $T$ completely semi-stable. For instance the tree of Figure 6 is stable, but is not semi-stable at $v$ by Theorem 2 .

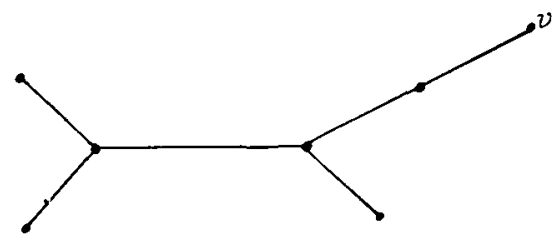

Figure 6

In conclusion we note that for graphs other than trees it is not possible to say that $G$ completely semi-stable implies $G$ stable. In Section 1 we saw that regular graphs are completely semi-stable, but in general they are not stable. Take, for instance, the cycle on $n$ vertices. Similarly the graphs $P_{m} \times P_{n}$ which are again completely semistable (for

(i) neither $m$ nor $n$ equal to 1 ,

(ii) if $m=2$ then $n=2$, and vice-versa,

(iii) $m$ and $n$ not both equal to 3 )

are not in general stable. In fact only $P_{2} \times P_{2}$ is stable.

\section{References}

[1] Frank Harary, Graph theory (Addison-Wesley, Reading, Massachussets; London; Ontario; 1969).

[2] D.A. Holton, "Stable trees", Proc. First Austral. Conf. Combinatorial Math., Newcastle, 1972, 15-21 (TUNRA, Newcastle, 1972).

[3] D.A. Holton, "Two applications of semi-stability", Discrete Math. 4 (1973), 151-158.

[4] D.A. Holton, "Some results on semi-stable graphs", Proc. British Combinatorial Soc. (Aberystwyth, 1973). 
[5] D.A. Holton, "A report on stable graphs", J. Austral. Math. Soc. 15 (1973), 163-171.

[6] D.A. Holton and Douglas D. Grant, "Regular graphs and stability", (submitted).

[7] Helmut Wielandt, Finite, permutation groups (translated by R. Bercov; Academic Press, New York, London, 1964).

Department of Mathematics,

University of Melbourne,

Parkville,

victoria. 